\title{
Monolithic Integration of a High Power Semiconductor Master Oscillator Power Amplifier
}

\author{
M. Faugeron, M. Vilera, A. Pérez-Serrano, J. M. G. Tijero, I. Esquivias , M. Krakowski and F. van Dijk
}

\begin{abstract}
We present a high power semiconductor Master Oscillator Power Amplifier monolithically integrated on InP, which includes a modulation section. This device can be used for random-modulation-continuous wave lidar systems or free-space communications.
\end{abstract}

Index Terms - Distributed feedback lasers, semiconductor optical amplifier, integrated optics devices, multi-section laser, master oscillator power amplifier.

\section{INTRODUCTION}

High power, single-mode semiconductor emitters at $1.55 \mu \mathrm{m}$ are very promising devices for lidar systems and freespace communications thanks to their low weight, compactness, high wall-plug efficiency and radiation hardness. To compete with fiber and solid-state lasers, they must exhibit output power $>0.5 \mathrm{~W}$ with a stable wavelength.

\section{DESIGN OF THE STRUCTURE}

We have investigated the Master Oscillator Power Amplifier (MOPA) architecture which usually consists of two sections, a distributed Bragg reflector (DBR) or distributed feedback (DFB) laser acting as a master oscillator and a power semiconductor optical amplifier (SOA). The MOPA architecture seems the more convenient solution to obtain both high power and single-mode operation [1], [2]. In that case, the SOA is often flared to increase its saturation power.

Instabilities in MOPA devices mainly comes from 2 factors: the disturbance of the DFB laser due to the light which returns into it, the mode competition between the whole cavity defined by the 2 chip facets and the DFB laser (Fig. 1). To mitigate these 2 effects, one key parameter is the reduction of the reflections at the SOA facet. To decrease as much as possible the reflections coupled to the waveguide mode, we have tilted the SOA waveguide and we have included a bent between the DFB laser and the flared SOA (Fig. 2). One advantage of the bent MOPA architecture is that we can both control the reflectivity of the DFB laser back facet (waveguide perpendicular with the facet) and have a tilt for the SOA waveguide. The bent section can be used as a pre-amplifier SOA or as a modulation section (forward-biased).

The MOPA chips were High Reflectivity (HR) / Anti
Reflectivity (AR) coated. Then individual chips were mounted p-side up on Aluminum Nitride submounts (Fig. 3).

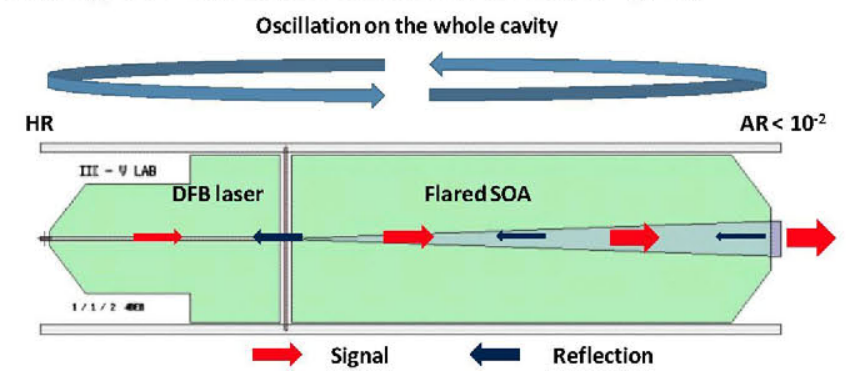

Fig. 1. Schematic of a straight MOPA.

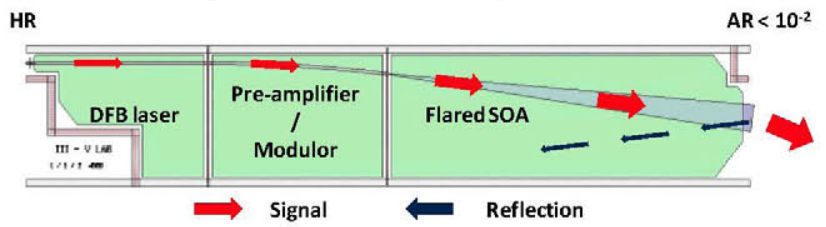

Fig. 2. Schematic of a bent MOPA.

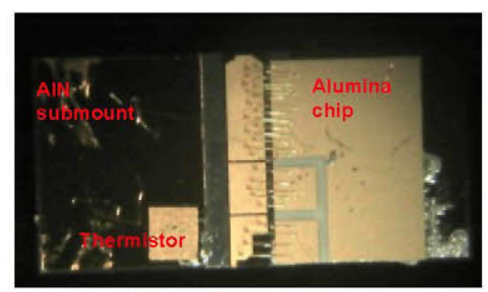

Fig. 3. Picture of the bent MOPA mounted on a AIN submount.

\section{RESULTS}

For all the devices shown in the letter, the DFB laser, the modulator and the flared SOA lengths are respectively 1,1 and $3 \mathrm{~mm}$. Fig. 4 is a plot of the output power as a function of the flared SOA bias current at $10^{\circ} \mathrm{C} \quad\left(\mathrm{I}_{\mathrm{DFB}}=400 \mathrm{~mA}\right.$, $I_{\text {mod }}=300 \mathrm{~mA}$ ). The maximum output power is $620 \mathrm{~mW}$ for 5.7 A. With shorter device (same DFB laser and modulator length but only $2 \mathrm{~mm}$ long flared SOA), we have obtained a maximum output power of 430,510 and $600 \mathrm{~mW}$ respectively at 18,12 and $6^{\circ} \mathrm{C}\left(\mathrm{I}_{\mathrm{SOA}}=3 \mathrm{~A}\right)$.

The optical spectrum is plotted Fig. 5 for $\mathrm{I}_{\mathrm{DFB}}=400 \mathrm{~mA}$, $I_{\text {mod }}=300 \mathrm{~mA}$ and $I_{S O A}=3 \mathrm{~A}$. The device is single-mode with a side mode suppression ratio (SMSR) superior to $45 \mathrm{~dB}$. The peak is blue-shifted $(17 \mathrm{~nm})$ compared with the amplified spontaneous emission. Be closer or slightly red-shifted from the maximum of the ASE will be better to get a higher output 
power. For an application point of view, the stability of the peak wavelength is very important. Fig. 6 shows the evolution of the optical spectra as a function of the DFB laser bias current for $\mathrm{I}_{\mathrm{mod}}=300 \mathrm{~mA}$ and $\mathrm{I}_{\mathrm{SOA}}=3 \mathrm{~A}$. Because the DFB laser is not $\lambda / 4$ phase-shifted there are 2 peaks close to the threshold (each side of the stop-band). From $\mathrm{I}_{\mathrm{DFB}}=200 \mathrm{~mA}$, the laser remains purely single-mode without any modehopping. The parabolic evolution of the wavelength is due to heating effects (standard for DFB laser).

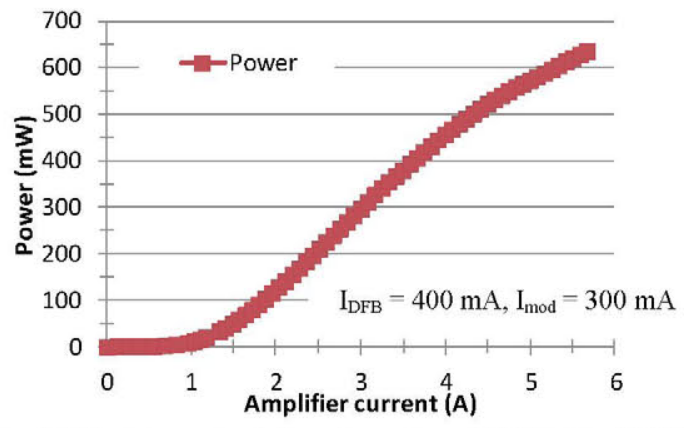

Fig. 4. P-I measurement for a $5 \mathrm{~mm}$ long $\mathrm{MOPA}\left(\mathrm{T}=10^{\circ} \mathrm{C}\right)$.

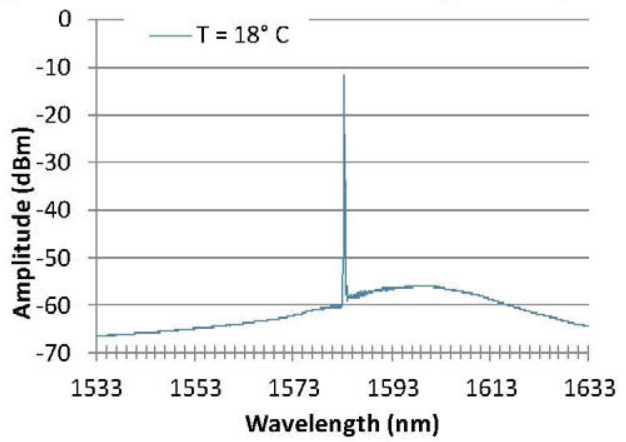

Fig. 5. Optical spectrum $\left(\mathrm{T}=18^{\circ} \mathrm{C}\right)$.

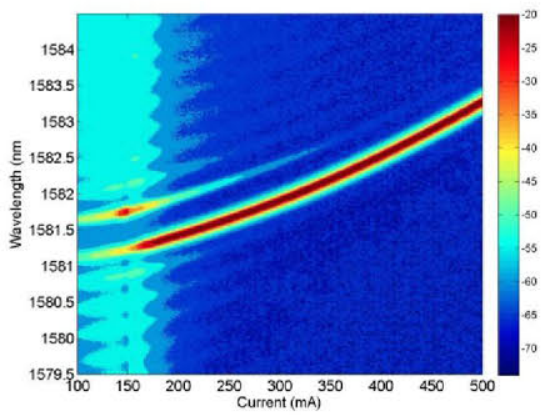

Fig. 6. Optical spectra (variation of $I_{D F B}, I_{m o d}=300 \mathrm{~mA}$ and $I_{S O A}=3 \mathrm{~A}$ ).

The optical linewidth measured using the delayed selfheterodyne method (1 km SMF fiber) was in the 2 to $5 \mathrm{MHz}$ range for high DFB bias currents. We have observed an increase of the linewidth with the increase of the flared SOA current (Fig. 7). We think this is due to an increase of the current source noise.

Fig. 8 shows preliminary modulation results of a similar device with different grating pitch leading to a slightly different emission wavelength and a lower maximum output power $(\sim 180 \mathrm{~mW})$. The $\mathrm{CW}$ bias conditions of this device are: $\mathrm{I}_{\mathrm{DFB}}=300 \mathrm{~mA}$ and $\mathrm{I}_{\mathrm{PA}}=2.5 \mathrm{~A}$. The response to the superimposition of a dc bias current $\mathrm{I}_{\mathrm{mod}}=100 \mathrm{~mA}$ and a 12.5 $\mathrm{MHz}$ square signal (peak-to-peak voltage $\mathrm{Vpp}=18 \mathrm{~V}$ ) applied to the modulation section (bent section) is shown in Fig. 8a. Fig. 8b shows the optical modulation amplitude (OMA) and the Extinction Ratio (ER) as a function of the modulation amplitude Vpp. At $12.5 \mathrm{MHz}$ a maximum OMA of $\sim 170 \mathrm{~mW}$ with an ER of $\sim 42 \mathrm{~dB}$ is obtained for $\mathrm{Vpp}=20 \mathrm{~V}$. This modulation results are encouraging for the application of the device as laser source for the detection of atmospheric $\mathrm{CO}_{2}$ by random modulation-continuous wave differential absorption lidar (RMCW DIAL) [3]. (a) $\mathrm{I}_{\mathrm{SOA}}=2.2 \mathrm{~A}$

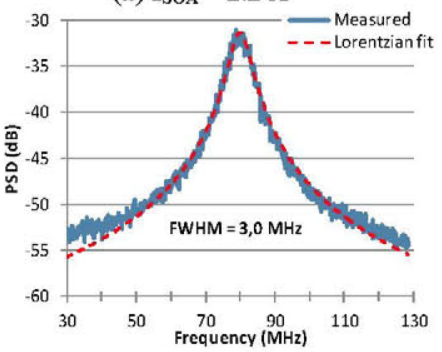

Fig. 7. Optical linewidth for I (b) $\mathrm{I}_{\mathrm{SOA}}=5.0 \mathrm{~A}$.

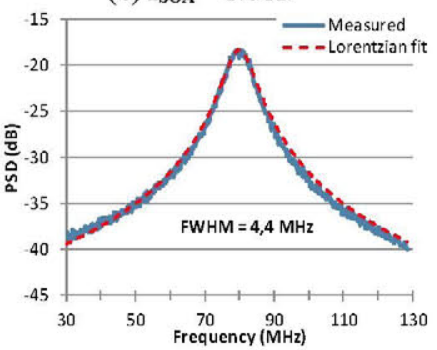

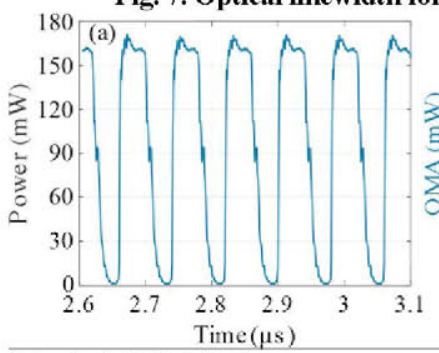

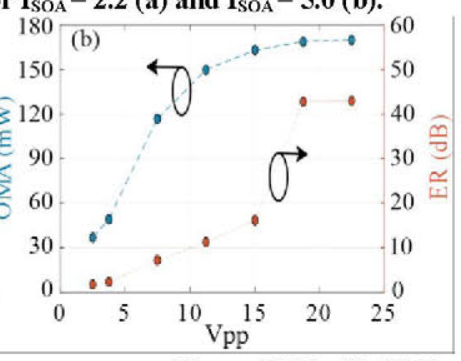

Fig. 8. (a) Modulation response to a square wave $(\mathrm{Vpp}=18 \mathrm{~V})$. (b) OMA and ER. $f=12.5 \mathrm{MHz}, I_{\mathrm{DFB}}=300 \mathrm{~mA}, I_{\mathrm{mod}}=100 \mathrm{~mA}$ and $I_{\mathrm{SOA}}=2.5 \mathrm{~A}$.

\section{CONCLUSION}

We have fabricated a 3-section monothically integrated MOPA. The output power is in the $400-600 \mathrm{~mW}$ range and the wavelength emission peak is single-mode (SMSR $>45 \mathrm{~dB}$ ) and stable. The optical linewidth is below $5 \mathrm{MHz}$. Preliminary modulation results indicate the suitability of these devices for RMCW lidar applications.

\section{ACKNOWLEDGMENT}

The authors acknowledge the European Commission Directorate-General for Research and Innovation within the framework of the European Space Project BRITESPACE under Grant 313200, the Communidad de Madrid within the Program SINFOTON-CM under Grant S2013/MIT-2790 and the Ministerio de Economia y Competitividad of Spain through the RANGER Project under Grant TEC2012-38864-C03-02.

\section{REFERENCES}

[1] M. L. Osowski, et al., "High-power semiconductor lasers at eye-safe wavelengths," in proc. SPIE, Laser Technol. Defense Secur. V, vol. 7325 , p. $73250 \mathrm{~V}$, May 2009.

[2] L. Hou, et al., "Narrow linewidth laterally coupled $1.55 \mu \mathrm{m}$ AlGaInAs/InP distributed feedback lasers integrated with a curved tapered semiconductor optical amplifier," Opt. Lett., vol. 37, no. 21, pp. 4525-4527, Nov. 2012.

[3] I. Esquivias, et al, "High-brightness all semiconductor laser at 1.57 $\mu \mathrm{m}$ for space-borne lidar measurements of atmospheric carbon dioxide: device design and analysis of requirements" in proc. SPIE 9135, Laser Sources and Applications II, 913516 doi:10.1117/12.2052191 (2014). 